\title{
Bruckner Red Light Reflex Test in a Hospital Setting
}

\author{
Saiju $R,{ }^{1}$ Yun $S,{ }^{2}$ Yoon PD, ${ }^{2}$ Shrestha $M K,{ }^{1}$ Shrestha UD ${ }^{1}$
}

${ }^{1}$ Tilganga Institute of Ophthalmology, Kathmandu, Nepal

${ }^{2}$ Faculty of Medicine, University of New South Wales, Sydney, Australial

\section{Corresponding Author}

Rohit Saiju

Tilganga Institute of Ophthalmology

Kathmandu, Nepal

Email: rsaiju@tilganga.com.np

\section{Citation}

Saiju R, Yun S, Yoon PD, Shrestha MK, Shrestha UD.Bruckner Red Light Reflex Test in a Hospital Setting. Kathmandu Univ Med J 2012;38(2):23-26.

\begin{abstract}
Background

Childhood blindness is a major problem in developing world including Nepal that needs to be addressed.
\end{abstract}

\section{Objective}

The objective of study was to measure the effectiveness of the Bruckner red reflex test for screening of posterior segment opacities in children.

\begin{abstract}
Methods
Patients aged less than five years who came to the Tilganga Institute of Ophthalmology, Kathmandu from March to August, 2009 were recruited in this cross-sectional descriptive study. Children were screened with the Bruckner red light reflex test in a dark room. Patients with abnormal Bruckner tests had their eyes dilated for further investigations. Abnormalities were recorded. The number of patients with an abnormal Bruckner test was recorded and correlated with their potential pathology.
\end{abstract}

\section{Results}

A total of 172 patients with 97 boys and 75 girls were included. Twenty-three had abnormal Bruckner test results in either one or both eyes. Four of these twentythree patients had no pathology diagnosed; hence the sensitivity of the Bruckner screen to diagnose significant pathology was calculated at $82.6 \%$. Of the 153 patients with normal results, two patients were found to have pathology detected, which included cataract and strabismus. Hence, the specificity of this test was $98.7 \%$. The screening test had a positive predictive value of $90.5 \%$ and a negative predictive value of $97.4 \%$.

\section{Conclusion}

Bruckner test screening is a sensitive and specific marker for detecting the posterior segment opacities in children. We recommend utilizing the test to evaluate these problems where other technologies are rare.

\section{KEY WORDS}

Nepal, Red flex test, Retinoblastoma, Screening

\section{INTRODUCTION}

It has been estimated that over 1.4 million children are blind in the world, with around three-quarters living in developing countries. The prevalence of childhood blindness varies from $0.3 / 1000$ in some developed countries, to $1.2 / 1000$ in poorer countries. ${ }^{1}$ This discrepancy in prevalence is likely due to the higher incidence of risk factors and reduced access to eye health services. In developing countries like Nepal, childhood blindness is a major public health problem. It is believed that more than $90 \%$ of blindness would be avoidable through access to ophthalmic health services and paediatric eye screening.

Screening and eye examination in a paediatric population is a crucial service to detect blinding eye diseases. The red reflex test aims to identify opacities in the visual axis and pathologies of the posterior segment. It diagnoses the common causes of childhood blindness that includes congenital cataract, undiagnosed amblyopia, retinoblastoma and corneal opacities. ${ }^{1}$

Red reflex screening has traditionally utilized the Bruckner test. Although newer tests, such as photo-screening have been introduced, the Bruckner test has the advantage of being simple, fast, cost effective and novel. ${ }^{2}$

The aim of study was to measure the effectiveness of the Bruckner red reflex test in the hospital setting and then screen posterior segment opacities in children. This helps to combat childhood blindness in Nepal, particularly in rural communities, where the problem is most significant. 


\section{METHODS}

The cross sectional, descriptive study was designed to recruit patients aged less than five years that came to the Tilganga Institute of Ophthalmology (TIO), Kathmandu with various complaints over a five months period (March 30th to August 15th, 2009). All consecutive cases were included during study period.

\section{Examination procedures}

Children were screened with the Bruckner red light reflex test in a dark room as follows. The ophthalmologist used a direct ophthalmoscope (HEINE Beta 200, Optotechnik, Germany) to examine the patients. The Bruckner test was carried out by holding an ophthalmoscope at a distance of one meter from the subject's eyes and focusing on the pupil to see if there is any asymmetry of color and brightness in the red reflexes of both eyes. In addition, a routine ophthalmic history and examination with slit lamp was performed by two experienced ophthalmologists. All patients were treated appropriately after diagnosis was reached.

Patients with abnormal Bruckner tests had their eyes dilated for further investigations. Abnormalities were recorded. The number of patients with an abnormal Bruckner test such as leukocoria, unclear, or opaque light reflex were recorded and correlated with their potential pathology.

Protocol was developed to collect the detail data of the children. Collected data were edited in the clinical site and followed by central editing. Edited data were coded, classified and entered into Microsoft Excel 2007 and analysis was done in SPSS version 11.5.

TIO Institutional Review Committee granted ethical approval for the research. Verbal consent for inclusion in this study was received from the parents and guardians before examination was commenced.

\section{RESULTS}

A total of 172 patients, which included 97 boys (56.4\%) and 75 girls (43.6\%) with mean age of $14.4 \pm 10.6$ months were assigned to this study. This included ninety-nine males with an average age of 14.7 months and seventy-seven females with an average age of 14.0 months. Patients displayed a variety of symptoms, most commonly, redness and watering

Table 1. Results of Bruckner test screening.

\begin{tabular}{llll|} 
Test\Result & $\begin{array}{c}\text { Positive Diag- } \\
\text { nosis (M:F) }\end{array}$ & $\begin{array}{c}\text { Negative Diag- } \\
\text { nosis (M:F) }\end{array}$ & Total (M:F) \\
\hline Abnormal Bruckner & $199: 10)$ & $4(3: 1)$ & $23(12: 11)$ \\
\hline Normal Bruckner & $2(1: 1)$ & $151(86: 65)$ & $153(87: 66)$ \\
\hline Total & $21(10: 11)$ & $155(89: 66)$ & $176(99: 77)$ \\
\hline
\end{tabular}

Table 2. Analysis of Bruckner test screening.

\begin{tabular}{llll}
\hline Abnormal & Total $(\mathbf{n = 2 3 )}$ & Normal & Total $(\mathbf{n = 1 5 3})$ \\
\hline Congenital Cataract & 9 & True negative & 151 \\
\hline Retinoblastoma & 4 & False negative & 2 \\
\hline False positive & 4 & $\begin{array}{l}\text { Note) False } \\
\text { negatives }\end{array}$ \\
\hline Strabismus & 1 & Cataract: 1 \\
\hline Nebular scar & 1 & \\
\hline $\begin{array}{l}\text { Enucleation } \\
\text { Congenital anoph- }\end{array}$ & 1 & Strabismus: 1 & \\
thalmos & 1 & \\
\hline $\begin{array}{l}\text { Refractive error/all } \\
\text { Conjunctivitis }\end{array}$ & 1 & \\
\hline \begin{tabular}{l} 
Hyphema \\
\hline
\end{tabular} & 1 & \\
\hline
\end{tabular}

of their eyes. Among those patients selected, twenty-three had abnormal Bruckner test results in either one or both eyes (table 1). Their eventual diagnoses included nine cataracts, four retinoblastomas, and one each of hyphema, asymmetric refractive error, congenital enophthalmos, nuclear scar, strabsimus and enucleation (table 2). Four of these twenty-three patients had no pathologies diagnosed; hence the sensitivity of the Bruckner screen was calculated at $82.6 \%$ (table 3).

Table 3. Statistical analysis of Bruckner screen.

\begin{tabular}{|ll|}
\hline Variables & Descriptive statistics \\
\hline No. of males & 99 \\
\hline No. of females & 77 \\
\hline Mean age \pm SD & $14.4 \pm 10.6$ months \\
\hline Sensitivity & $82.6 \%$ \\
\hline Specificity & $98.7 \%$ \\
\hline Positive predictive value & $90.5 \%$ \\
\hline Negative predictive value & $97.4 \%$ \\
\hline
\end{tabular}

One hundred and fifty-three patients had normal Bruckner test results. Of these, one hundred and fifty-one patients presented with pathologies undetectable by Bruckner test screening such as congenital naso-lacrimal duct obstruction and conjunctivitis. However, two patients were found to have pathologies normally detectable using the Bruckner test, which included one cataract and one strabismus case (table 2). Hence, the specificity of the Bruckner screening for a pathological diagnosis was $98.7 \%$.

The screening test had a positive predictive value of $90.5 \%$ and a negative predictive value of $97.4 \%$ (table 3). Eleven females were diagnosed with detectable pathologies. Of these, ten had an abnormal Bruckner test. Ten males had detectable pathologies, with nine having abnormal Bruckner tests.

\section{DISCUSSION}

The Bruckner red light reflex screen was first described 
in 1962 as a means of screening for the amblyogenic risk factors of anisometropia, strabismus or media opacity. ${ }^{3}$ Its utility has now been accepted and commonly practiced in ophthalmic screening all over the world. The high sensitivity and specificity from our study suggest that the Bruckner test can be used as an effective screen for visual defects in a paediatric population.

In the late 1990s, a new arm in red reflex screening was introduced, namely photo-screening. This technique involves the use of a photographic image of the pupillary red reflexes of a patient and evaluation of the photographs by the practitioner. Its main advantage over the traditional Bruckner test is that it does not require a compliant patient to stay still and is not restricted by time for analyzing the photos. ${ }^{4}$ Carrera et al reported high sensitivity (82\%), specificity (91\%), and accuracy (84\%) using photo-screening to detect amblyopia in a paediatric population. ${ }^{5}$ For these reasons, photo-screening is now widely employed in developed countries.

However, in less developed countries, such as Nepal, the Bruckner test offers numerous advantages. Firstly, the test is considerable cheaper compared to photoscreening test which is reported to cost up to US\$10 for each patient examined. ${ }^{6}$ The Bruckner test is a readily reproducible test, merely requiring a dark environment and an ophthalmoscope. The test is also simple as persons with no or minimal experience can be trained easily to perform screening. Further, it can potentially screen a large population in a short period of time, with the average time reported for each eye being ten seconds. ${ }^{7}$ Finally, our results showed that the test has high sensitivity $(82.6 \%)$ and specificity (98.7\%).

There are some limitations of our study. The selection criteria were both pre-verbal and verbal infants under five years of age who attended TIO for treatment of their various symptoms. This meant that some infants were able to voice their symptoms whilst others relied on their parents to provide their history. Moreover, TIO is a large, busy, urban hospital in Nepal's largest city, Kathmandu. Therefore, the subjects chosen were not randomly selected, controlled and representative of the whole Nepali population. This is particularly important as the majority of eye diseases occur in the rural Nepalese setting, and much of our proposal is targeted at this population.

In addition, we found that there were three cases of strabismus missed with red light reflex screening, although they were ultimately diagnosed through routine history and examination by the ophthalmologist. On further analysis of the three cases, we found that intermittent alternate divergent squint was a difficult condition to diagnose with the Bruckner test due to its severity. Another case of left/ alternate esotropia was not ascertained by the Bruckner test, as reflexes were symmetrical in both eyes. Therefore, only one patient with esotropia of 30 prism dioptres was found out to be the 'true' false negative. These cases illustrate that the Bruckner test has potential fallacies when diagnosing relatively less severe or symmetrical strabismus.

The high sensitivity and specificity obtained may partly be explained by the vast experience of the ophthalmologists. Examination by less experienced persons would likely produce less spectacular results. Intra-observer and interobserver variations will undoubtedly occur. Despite these limitations, our study has displayed that the Bruckner test is an effective and suitable screen in identifying conditions such as cataracts, severe strabismus and ocular tumors in the developing world.

The current American Academy of Pediatrics Policy recommends that every child should be receiving red light reflex screening from a pediatrician or a primary healthcare physician within their first two months of life. ${ }^{8}$ This model involves highly qualified and experienced healthcare workers and aims to filter avoidable ophthalmic conditions in a two leveled approach; initial examination by the primary care person and further examination by specialized personnel in positively tested patients. The model ensures high risk groups (family history or previous ophthalmic/ medical history) receive extra attention by referring them directly to ophthalmic specialists.

Adapting this American model to Nepal is difficult due to its vastly different health care system and population. Nepal is a developing country with only eighteen eye hospitals and eight paediatric ophthalmologists to cover a population of twenty-eight million people. Moreover, Nepal has a relatively young population, with the annual growth rate in excess of two percent and $36.6 \%$ of the total population less than 16 years of age. ${ }^{9}$

Currently, there is no recognized national policy for paediatric ophthalmic screening in Nepal. We suggest a three-tier model whereby infants would first be screened using the Bruckner test by locally trained examiners including volunteers and local healthcare assistants at community health centers as vast majority of community health centers in Nepal are already fully equipped to perform the Bruckner test. Any patients with abnormal results should be sent to a local healthcare professional to be carefully consulted and examined. We believe that history and examination with an ophthalmoscope aided with dilatation will allow diagnosis and initiation of treatment for most pediatric eye conditions at this level. When screening by health care professional renders suspicious results they should be referred to a tertiary eye center where they are managed by an experienced ophthalmologist (fig.1). To ensure success with this model, much effort should be directed in training local healthcare professionals to use an ophthalmoscope and take an appropriate history. A pilot study looking at the accuracy of Bruckner testing by paramedics and volunteers in a rural Nepalese setting would shed much light on how much training is needed. Close liaison amongst the three tiers 
of healthcare workers is crucial. However, the greatest obstacle that needs to be overcome is ensuring that the targeted population, in particular the rural community, has access to this screening program. Clearly, a grass roots approach aimed at equipping local communities to do initial screening is mandatory in ensuring success.

\section{REFERENCES}

1. Gilbert C. New issues in childhood blindness. Community Eye Health 2001;14:53-56.

2. Tongue AC, Cibis GW. Bruckner test. Ophthalmology 1981;88:10411044.

3. Bruckner R. Exakte strabismus diagnostik bei1/ 2-3 jhrigen klindern miteinem einfachem verfahren, dem "Durchieeuch tungtest". Ophthalmologica 1962;144:184-98.

4. Paysse EA, Williams GC, Coats DK. Detection of Red Reflex Asymmetry by Paediatric Residents Using the Bruckner Reflex Versus the MTI Photoscreener. Paediatrics 2001; 108:74-81.

5. Carrera A, Saornil MA, Zamora MI. Detecting amblyogenic diseases with the photographic Bruckner test. Strabismus 1993; 1:3-9.

\section{CONCLUSION}

Bruckner test screening is a sensitive and specific marker for detecting opacities in the visual axis and pathologies of the posterior segment in children. We recommend a three-tier model, initially utilizing the Bruckner test at the community level, to combat the problem of childhood blindness in countries like Nepal where latest technologies like photo screening are rarely available.

6. Arnold RW, Armitage MD, Gionet EG. The Cost and Yield of Photo screening: Impact of Photo screening on Overall Paediatric Ophthalmic Costs. J Ped Ophthalmol \& Strabismus 2005; 42:103-11.

7. Kothari MT. Can the Bruckner test be used as a rapid screening test to detect significant refractive errors in children? Indian J Ophthalmol 2007;55:213-5.

8. American Academy of Paediatrics. Policy statement: Red reflex examination in infants. Paediatrics 2002; 109:980-81.

9. CIA World Fact Book 2003. Available from https://www.cia.gov/ library/publications/the-world-factbook/index.html on 1st of March, 2010. 